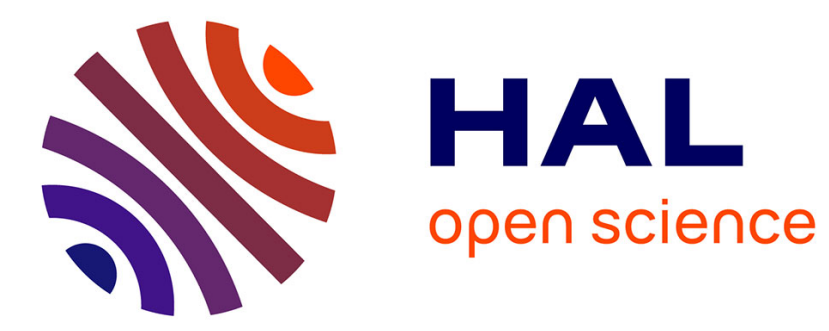

\title{
Core tungsten transport in WEST long pulse L-mode plasmas
}

X. Yang, P. Manas, C. Bourdelle, J. Artaud, R. Sabot, Yann Camenen, J. Citrin, F. Clairet, C. Desgranges, P. Devynck, et al.

\section{- To cite this version:}

X. Yang, P. Manas, C. Bourdelle, J. Artaud, R. Sabot, et al.. Core tungsten transport in WEST long pulse L-mode plasmas. Nuclear Fusion, 2020, 60 (8), pp.086012. 10.1088/1741-4326/ab9669 . hal-02989885

\section{HAL Id: hal-02989885 \\ https://hal.science/hal-02989885}

Submitted on 5 Nov 2020

HAL is a multi-disciplinary open access archive for the deposit and dissemination of scientific research documents, whether they are published or not. The documents may come from teaching and research institutions in France or abroad, or from public or private research centers.
L'archive ouverte pluridisciplinaire HAL, est destinée au dépôt et à la diffusion de documents scientifiques de niveau recherche, publiés ou non, émanant des établissements d'enseignement et de recherche français ou étrangers, des laboratoires publics ou privés. 


\title{
Core tungsten transport in WEST long pulse L-mode plasmas
}

\author{
X. Yang, ${ }^{1,2}$ P. Manas, ${ }^{3}$ C. Bourdelle, ${ }^{3}$ J. F. Artaud, ${ }^{3}$ R. Sabot,${ }^{3}$ Y. Camenen, ${ }^{4}$ J. Citrin, ${ }^{5}$ F. Clairet ${ }^{3}$ C. \\ Desgranges, ${ }^{3}$ P. Devynck, ${ }^{3}$ T. Dittmar, ${ }^{6}$ A. Ekedahl, ${ }^{3}$ N. Fedorczak, ${ }^{3}$ C. Gil,${ }^{3}$ T. Loarer, ${ }^{3}$ Ph. Lotte ${ }^{3}$ O. Meyer, ${ }^{3}$ \\ J.Morales, ${ }^{3}$ M. Peret, ${ }^{3}$ Y. Peysson, ${ }^{3}$ C. D. Stephens, ${ }^{7}$ G. Urbanczyk, ${ }^{8}$ D. Vézinet ${ }^{3}$ L. Zhang, ${ }^{1}$ and X. Gong ${ }^{1}$ \\ ${ }^{1}$ Institute of Plasma Physics, Chinese Academy of Science, Hefei, 230031, China/P.R. China \\ ${ }^{2}$ University of Science and Technology of China, Hefei, 230026, China/P.R. China \\ ${ }^{3}$ CEA, IRFM, F-13108 Saint Paul-Lez-Durance, France \\ ${ }^{4}$ CNRS, Aix-Marseille Univ., PIIM UMR7345, Marseille, France \\ ${ }^{5}$ DIFFER - Dutch Institute for Fundamental Energy Research, Eindhoven, the Netherlands \\ ${ }^{6}$ Forschungszentrum Jülich GmbH, Institut für Energie- und Klimaforschung-Plasmaphysik, D-52425 Jülich, Germany \\ ${ }^{7}$ University of California, Los Angeles, California 90095-1547, USA \\ ${ }^{8}$ Advanced Energy Research Center, Shenzhen University, Shenzhen 518060, China
}

\begin{abstract}
Tungsten transport is investigated in WEST long pulse L-mode plasmas operated with the strike point on the actively cooled upper tungsten divertor. The pulses are mostly heated by lower hybrid waves. It is experimentally found that tungsten does not centrally accumulate throughout these $\sim 30$ s reproducible discharges despite large normalised electron density gradients $R / L_{n_{e}}$. To explain these observations, turbulent and neoclassical transport of electrons and tungsten ions are computed with GKW [1] and NEO [2,3] respectively. Additionally, interpretative integrated modelling simulations are also performed to keep data coherency despite the lack of measurements of some quantities such as the $T_{i}$ profiles. Modelled $R / L_{n_{e}}$ are found consistent with interferometry inversions and the tungsten peaking factor $R / L_{n_{W}}$ remains comparable to $R / L_{n_{e}}$ due to dominant turbulent diffusivities inside $r / a=0.3-0.8$. In the central region $r / a<0.3$ neoclassical $\mathrm{W}$ transport dominates but the convective velocities are several order of magnitudes lower compared to plasmas with toroidal rotation velocities induced by a neutral beam injection (NBI) torque. Finally, nitrogen is seeded in these pulses leading to an enhanced energy content which is consistent with stabilised ion temperature gradient modes from dilution.
\end{abstract}

PACS numbers:

\section{INTRODUCTION}

Tungsten (W) accumulation in the core of tokamak plasmas causes a large increase of the radiated power due to its high cooling rate, which severely restricts the operational domain. For instance, it is routinely observed in machines with tungsten environments such as JET and ASDEX Upgrade, that $\mathrm{W}$ can accumulate in neutral beam injection (NBI) only heated $\mathrm{H}$-mode plasmas $[4,5]$. Indeed NBI torque and central particle sources lead to enhanced inward tungsten convection. In this context, the tokamak WEST, which operates in a full W environment $[6,7]$, has achieved $32 \mathrm{~s}$ L-mode pulses without any sign of $\mathrm{W}$ accumulation. These pulses were performed on the upper actively cooled $\mathrm{W}$ divertor [8] with nitrogen puffing phases in order to explore the ammonia formation [9]. Observations of the absence of $\mathrm{W}$ accumulation with only radio-frequency heating systems (no input torque) are not new, e.g. [13] where H-mode plamas with only ion cyclotron resonance heating (ICRH) are analysed. Nevertheless, for these Alcator C-Mod plasmas, the density profiles are flat and the ICRH heating systems are known to prevent $\mathrm{W}$ accumulation [14]. Thus, the interesting points raised by these WEST long pulse L-mode plasmas are the fact that no ICRH has been used (no beneficial effect on $\mathrm{W}$ transport) and that the density profiles are particularly peaked, which should lead to unfavourable $\mathrm{W}$ convection. Therefore, it is important to understand tungsten transport in these conditions for future optimized operations.

Core W transport results of both neoclassical (collisional) and turbulent physics [10, 11]. Neoclassical particle convection is proportional to the normalised density and ion temperature gradients. Hence electron density peaking plays a key role in $\mathrm{W}$ accumulation. In the presence of toroidal rotation, the detrimental effect of density peaking is further amplified [12]. On the other hand, microinstabilities such as the ion temperature gradient (ITG) and the trapped electron mode (TEM) also produce significant core impurity transport, which with sufficiently large turbulent diffusion can compensate for the unfavourable neoclassical convection. In this contribution, WEST long pulse L-mode plasmas are studied where despite peaked electron densities, the W concentration remains stable throughout the whole pulse. Tungsten transport analysis are performed for a representative case thanks to the neoclassical code NEO $[2,3]$ and to the gyrokinetic codes GKW [1] and QuaLiKiz [15]; QuaLiKiz employs a high-frequency expansion for the eigenfunction evaluation, enabling faster computations compatible with integrated modelling.

The paper is organised as follows. In Section 2 the WEST tokamak and the long pulse scenarios are described and in particular a representative pulse is further scrutinised. Soft X-ray (SXR) measurements to account for the $\mathrm{W}$ content are discussed and reflectometry measurements allow us to assess the turbulent state of the 
plasma, such as whether trapped electron mode turbulence is dominant or not. Additionally, stringent tests for data coherency, such as enforcing a consistent $Z_{\text {eff }}$ with respect to the flux consumption in ohmic phases, are performed with the transport code METIS [16]. These interpretative integrated modelling simulations are mandatory to compensate or minimise the lack of experimental data due to the very recent operation of WEST. In Section 4, modelling tools are described and used to analyse the main experimental observations from Section 2 such as the peaked electron density profiles, flat tungsten densities and improved confinement with nitrogen seeding. Finally, conclusions are given in Section 5.

\section{EXPERIMENTAL SETUP AND OBSERVATIONS}

WEST is a full tungsten (W) superconducting actively cooled tokamak with a plasma volume of $14 \mathrm{~m}^{3}$, a major radius of $2.5 \mathrm{~m}$, an aspect ratio $A=R / r$ between 5 and 6 and a magnetic field of $3.7 \mathrm{~T}$. The WEST programme aims at power/particle exhaust studies on the ITER-like actively cooled tungsten divertor for discharges reaching 1000 s, while maintaining good bulk plasma performances. It is equipped with $9 \mathrm{MW}$ of ion cyclotron resonance heating $(\mathrm{ICRH})$ and $7 \mathrm{MW}$ of lower hybrid current drive (LHCD) $[6,7]$.

The upper divertor is made of actively cooled, Wcoated, copper tiles $[6,8]$. In the present phase 1 of WEST, the lower divertor is equipped with a set of actively cooled ITER-like plasma facing units (PFU) [17] and is complemented with inertially cooled $\mathrm{W}$ coated units. Other side limiters are all W coated (Fig. 1). In the 2nd phase of WEST operation, to be started in 2020, the lower divertor will be fully equipped with ITER-like PFUs [18].

The main plasma configuration is in lower single null (LSN), but WEST can be operated in double null (DN) as well as in upper single null (USN) to, in particular, perform long pulses on the actively cooled upper divertor in this first phase of operation. Thanks to reproducible 30 s pulses, 20 minutes of plasmas were achieved in 2 days in USN. Since these long pulses were performed to study ammonia formation, $N_{2}$ was puffed either from the upper divertor or the midplane. Boronisations [19] opened up the operational window to higher density ranges, but the fraction of radiated power remained around $50 \%$ of the LHCD power [20]. The reported experiments have been carried out 2 months after the last boronisation. This means that the short term potential effects on the plasma performances had fully vanished whilst the level of oxygen was lower than prior to the boronisation.

In this work, a USN $33 \mathrm{~s}$ long L-mode plasma with dominant electron heating and low torque is presented (discharge \#54178). To monitor the $\mathrm{W}$ behaviour, bolometers, SXR, UV spectroscopy and visible spectroscopy are installed which can be used to measure the

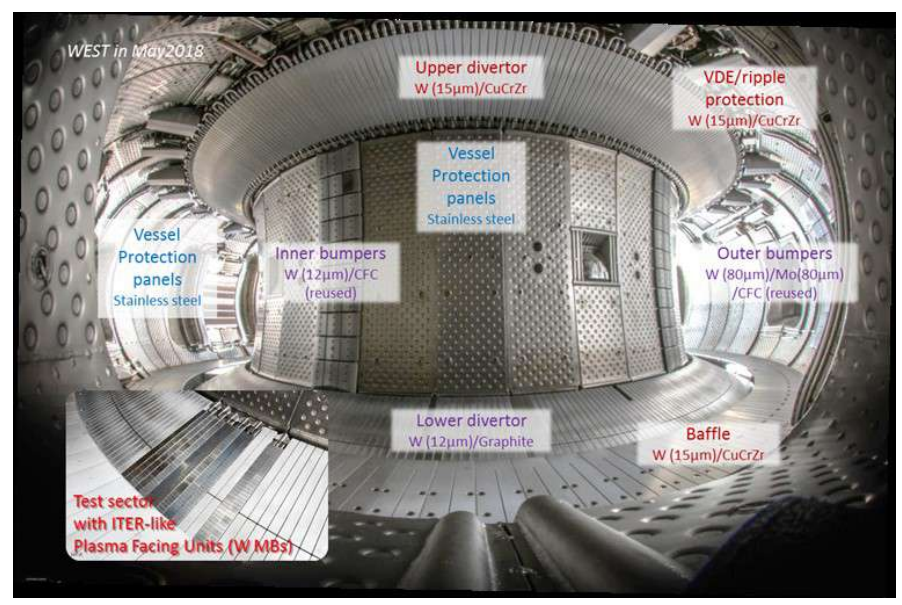

FIG. 1: Picture of WEST vacuum vessel, May 2018

radiated power, radiation of soft $\mathrm{X}$ emission, and the line emission of highly ionized W as well as W I, separately. Line of sights (LOS) of the SXR and a few among the 240 LOSs of the visible spectroscopy [21] are shown in Figs. 2(a) and (b), respectively.

\subsection{Absence of tungsten accumulation with peaked electron density profiles}

Time traces of several parameters of this representative WEST long pulse L-mode plasma are presented in Fig. 3. The magnetic field was at its nominal value, 3.7 $\mathrm{T}$, the plasma current at $0.4 \mathrm{MA}$ and the edge safety factor at $q_{95}=4.8$. Through the discharge heated via LHCD antennas with up to $2.8 \mathrm{MW}$, the radiated power, the peaking factor (defined as ratios of SXR line integrated intensities) and the UV line emission of W XLIV display a stable behaviour, which suggests that the W content remains constant. A significant increase in the radiated power is observed during the ICRH phase and is not further discussed in this paper. More details on the plasma response in presence of ICRH are reported in [23]. 

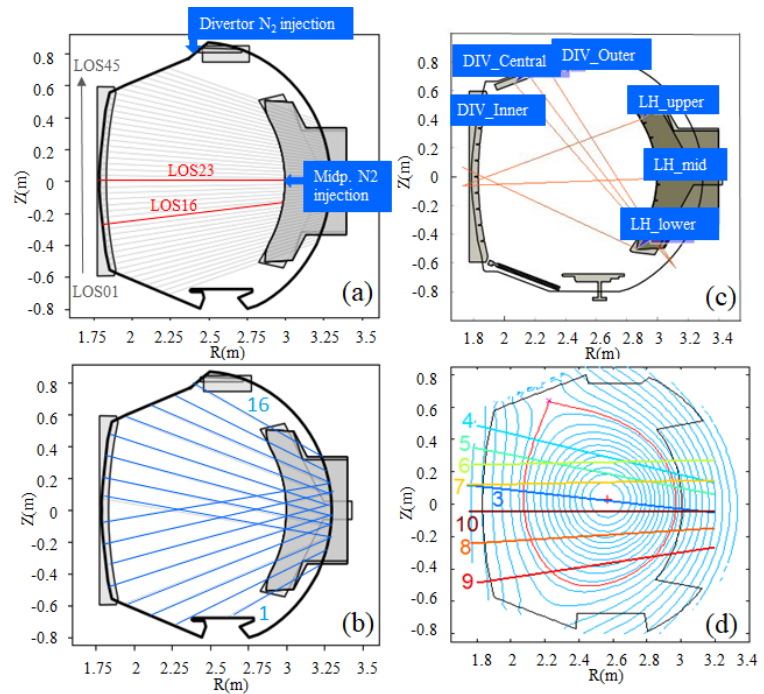

FIG. 2: (a) LOSs (gray lines) of SXR and the positions where the $N_{2}$ can be seeded. The two red LOSs of SXR will be used to define the $\mathrm{W}$ peaking factor. (b) LOSs of bolometers. (c) A few of the 240 LOSs of the visible spectroscopy diagnostic. (d) LOS of interferometers [22].

During the $N_{2}$ seeding phase, the plasma's stored energy and the neutron flux both increase, indicating improved confinement. Meanwhile, the SXR peaking factor and the UV line emission of W XLIV decrease and the total radiated power slightly increases. These observations are robustly reproduced in several other similar USN long pulses. Finally, it is to be noted that the $\mathrm{W}$ sources (not shown here) from the divertor and the LCHD limiter, monitored with visible spectroscopy (W I at $400.8 \mathrm{~nm}$ ), do not feature significant changes throughout the LHCD heated phase.

The electron temperature profiles are measured by electron cyclotron emission (ECE) as shown in Fig. 6 for the discharge \#54178 at $8 \mathrm{~s}$. $T_{e}$ is reliable in the range of $r / a<0.6$ when LHCD heating is used. Once beyond this range, the ECE signal is polluted by LHCD non-thermal electrons [24]. These suprathermal electrons can also affect the high field side signals yielding horizontal asymmetries on the $T_{e}$ profiles. For this reason, only the low field side (LFS) values are considered. The electron density profiles were inverted from interferometry measurements, with 8 lines of sight and using a modified hyperbolic tangent formulation [25]. These inverted profiles are compared to reflectometer measurements in Fig. 4 (top panel). During LHCD phases, stray ECE radiation is emitted by the fast electrons and deteriorates the signal to noise ratio. As a consequence the uncertainty on the profile positions from reflectometry rises from $\pm 1 \mathrm{~cm}$ to approximately $\pm 4 \mathrm{~cm}$. This issue, present in the current analysis, has been solved later by increasing the sweeping time from 1 to $10 \mu \mathrm{s}$; this reduces the frequency bandwidth on the signal detection in order to diminish the ECE noise. At the plasma's edge, density profiles measured by these two different diagnostics are consistent and feature a relatively large density peaking. This density peaking remains large even at mid-radius. Such peaked density profiles can lead to large inward neoclassical convective tungsten velocities and potentially to tungsten accumulation. Hence, to properly predict tungsten transport, theoretical models should first correctly predict these levels of electron density peaking. This is further investigated in Section 4. Finally, because some experimental data is lacking due to diagnostics being not available at the time of the WEST operation (e.g. $T_{i}$ profiles), a particular effort is carried out throughout this work to maximise the data consistency checks in order to minimize the impact of missing measurements. Interpretative integrated modelling is used together with synthetic diagnostics to maximise the data consistency validation. This methodology is described in Section 3.

\subsection{Density fluctuation measurements and TEM turbulence}

Density fluctuations were also measured with the core $\mathrm{X}$-mode reflectometer [26]. Measurements were performed at $t=3.7 \mathrm{~s}$, just before the LHCD ramp-up, and at $t=8 s$, LHCD plateau, before the $N_{2}$ injection phase. Each of the 2 channels probed 10 frequencies (radial positions). Each frequency plateau lasted $25 \mathrm{~ms}$, leading to an overall measurement time of $300 \mathrm{~ms}$. The positions of the probing zone were recovered using the density profiles provided by the interferometry lines inversion. The frequency spectra exhibit a classical shape with low frequency and broadband components [27, 28]. Unlike the Ohmic phase, the LHCD phase contained an increase of 15-20 dB of the background ECE noise. As shown in Fig. 5 a quasi-coherent component $[27,29]$ can be observed at around $70 \mathrm{kHz}$ with $r / a=0.37$ and $r / a=0.26$ on the LFS. Its amplitude is much smaller at $r / a=0.45$ and hardly above the broadband component further out. As it was shown in $[30,31]$, the quasi-coherent modes in the core are a signature of TEM turbulence. In the Ohmic phase, quasi-coherent modes are hardly detectable even if the amplitude of the broadband component is few $\mathrm{dB}$ lower.

\subsection{Improved energy content during $N_{2}$ seeding}

Improved confinement with impurity seeding is commonly observed in stellarators [32] and medium sized tokamaks whether, it is due to reduced core heat diffusivities and increased central electron densities for the radiative improved mode at TEXTOR-94 [33] and also for L-mode plasmas as in DIII-D [34], or due to improved pedestal pressure in $\mathrm{H}$-mode plasmas as in ASDEX Upgrade [35] and JT-60U [36]. For WEST discharge $\# 54178, N_{2}$ was puffed at a rate of $0.2 \mathrm{~Pa} \mathrm{~m}^{3} \mathrm{~s}^{-1}$ through the midplane between 10 and $20 \mathrm{~s}$ (see Fig. 3). 


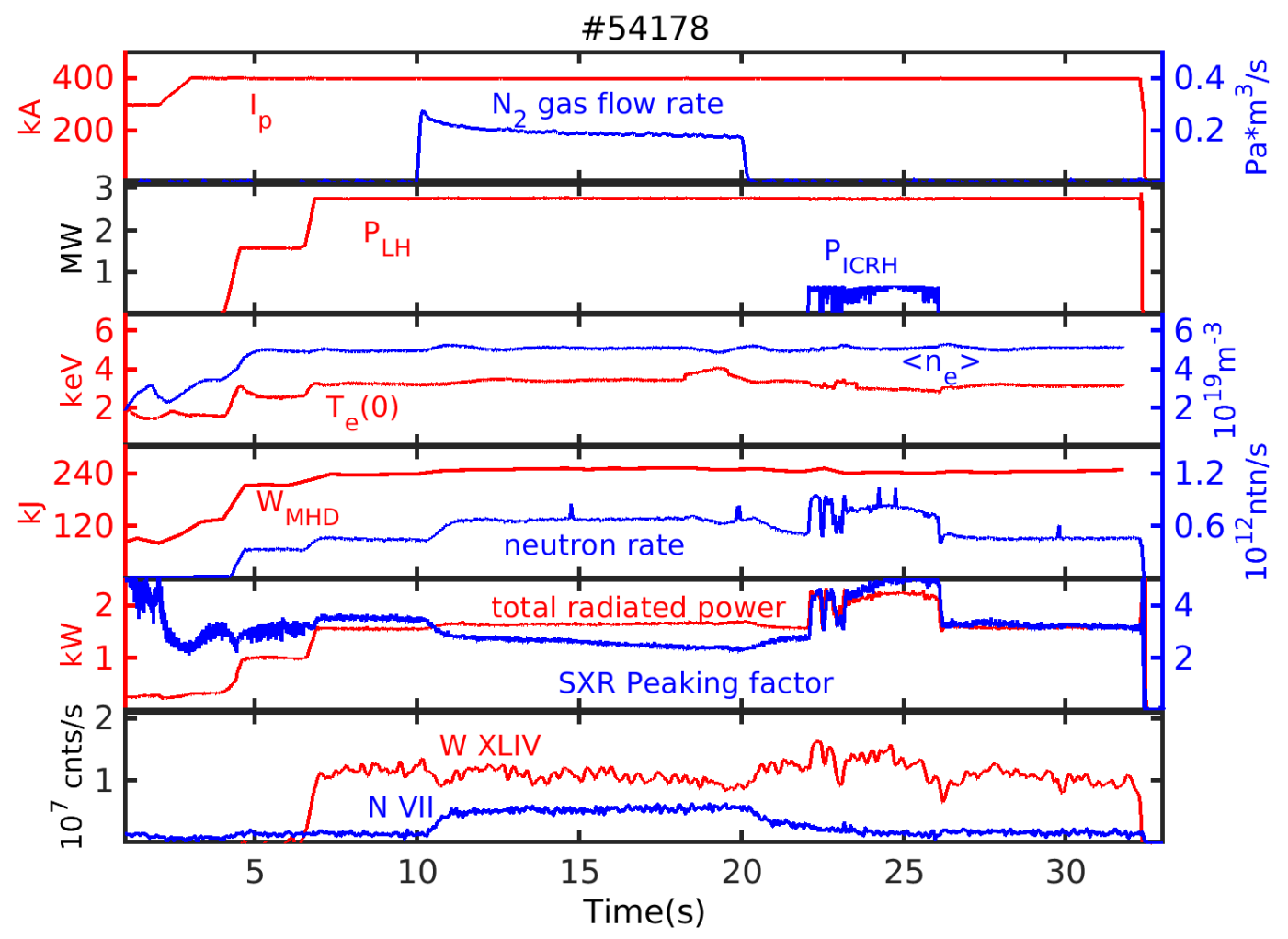

FIG. 3: From top to bottom, time traces of the plasma current and the $N_{2}$ gas flow rate from the midplane, heating powers, central $T_{e}$ and volume averaged $n_{e}$, plasma's stored energy $W_{\mathrm{MHD}}$ and the neutron flux, total radiated power and SXR peaking factor defined as $(I 23 / \mathrm{Ne}(\rho=0) / L 23) /(I 16 / \mathrm{Ne}(\rho=0.4) / L 16)$, with I the line integrated SXR intensity and L the length of the SXR LOS, line emission of W XLIV and N VII.

The plasma's stored energy and the thermal DD neutron flux increase by $5 \%$ and $50 \%$, respectively, indicating improved confinement and increased central ion temperature. The electron temperature profile and the line averaged electron densities are compared during phases with and without $N_{2}$ seeding in Figs. 4 (bottom panel) and 6 . It is found that these quantities, namely $n_{l}$ (in particular the line of sight looking at the most central region) and $T_{e}$, are identical within error bars before and during the $\mathrm{N}_{2}$ seeding phase. The improved confinement with $N_{2}$ seeding is robustly observed for several long pulses with similar injected powers $\left(P_{L H C D}=2.5-2.8 \mathrm{MW}\right)$, central electron density and temperature and magnetic field geometry, whether $N_{2}$ is injected from the upper divertor or the mid-plane. In Fig. 7 the increment in the DD neutron rate between phases with and without $N_{2}$ seeding for 10 discharges is compared to the increment in the normalised UV line emission of N VII. For 6 of these pulses $N_{2}$ was puffed from the upper divertor (\#54166 \#54164 \#54163 \#54160 \#54159 \#54152) and the rest from the mid-plane (\#54178 \#54177 \#54173 \#54170). A clear increase in the neutron rate is observed with the increased N VII line emission intensity normalised to the electron density, despite the plasma dilution. Such an increase of the neutron rate is the signature of increased core deuterium temperature.

The radiation pattern is also analysed by comparing the cases with and without $N_{2}$ seeding. It is observed in Fig. 8, by substracting the SXR emission profile after and before $N_{2}$ seeding, that the difference between these two phases is hollow. This is consistent with the reduction of the SXR peaking factor during the $N_{2}$ injection shown in Fig. 3. An increased off-axis SXR signal can be due to $\mathrm{N}_{2}$ emission and/or a modified W profile. The bolometry lines of sight show an increased radiated power mostly due to increased edge LoSs (the ones looking at the upper divertor) whereas the most central LoSs slightly decrease (see Fig. 8). The impact of $N_{2}$ seeding on the tungsten transport and the improved confinement is modeled and discussed further in Section 4 . 

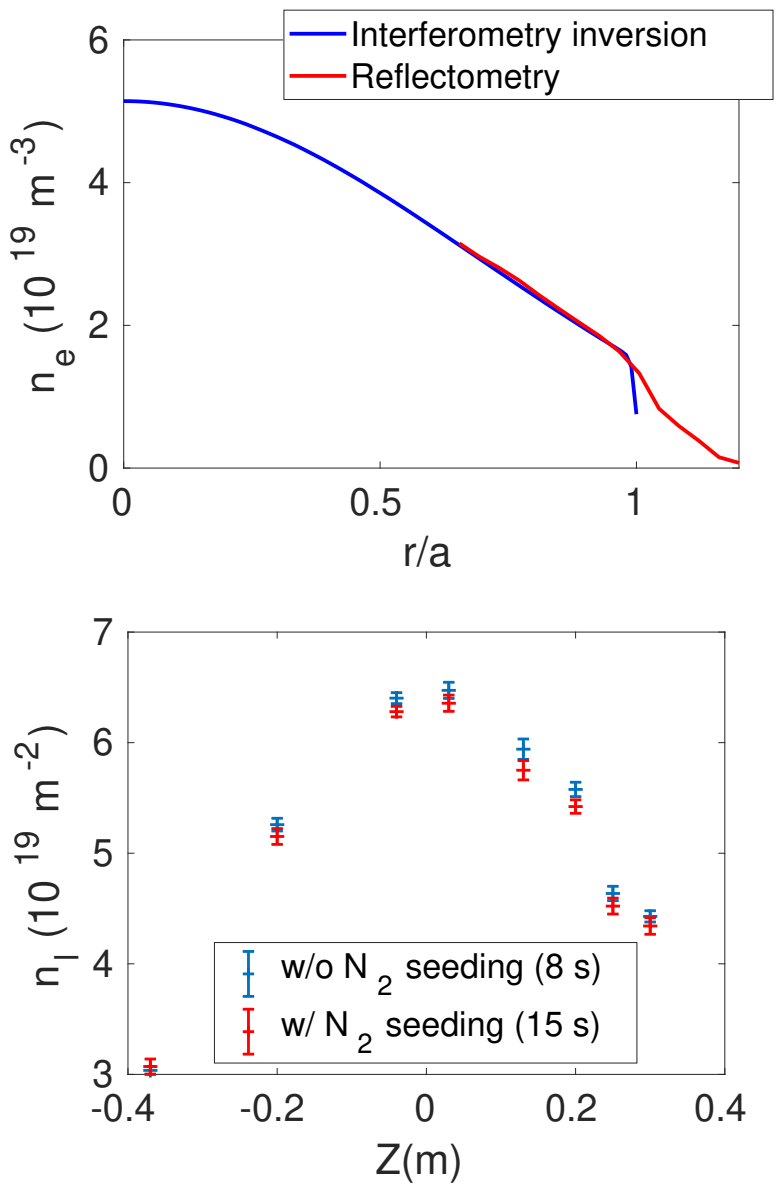

FIG. 4: (top panel) Inverted electron density profile from interferometry (blue) compared to the measured electron density profile from reflectometry (red) at $8 \mathrm{~s}$ for discharge \#54178. (bottom panel) Line integrated densities from interferometry for the phases with and without nitrogen seeding (phases at 8 and $15 \mathrm{~s}$ respectively) against the vertical coordinate $\mathrm{Z}$ given by the intersection of the line of sights with the vertical axis at $R=2.5 \mathrm{~m}$. Error bars are computed from variations within a $200 \mathrm{~ms}$ time window around the stationnary phases at 8 and $15 \mathrm{~s}$.

\section{INTEGRATED MODELLING AND DATA CONSISTENCY USING METIS}

We perform interpretative integrated modelling using METIS [16] to compensate the lack of measurements inherent to newly operating tokamaks as is the case for WEST. METIS is a fast integrated tokamak modelling tool combining 0D scaling laws for kinetic profiles with 1D current diffusion modelling and 2D equilibria. These simulations allow data consistency checks, such as central $T_{i}$ consistent with the measured DD neutron rate. Here, the electron temperature and density profiles used are fitted from ECE measurements and interferometry inversions (see Fig. 4 and 6).

In the USN of WEST, Bremsstrahlung LoSs are polluted by visible light reflections on the upper divertor. Therefore, the effective charge $\left(Z_{\text {eff }}\right)$ is deduced from the flux

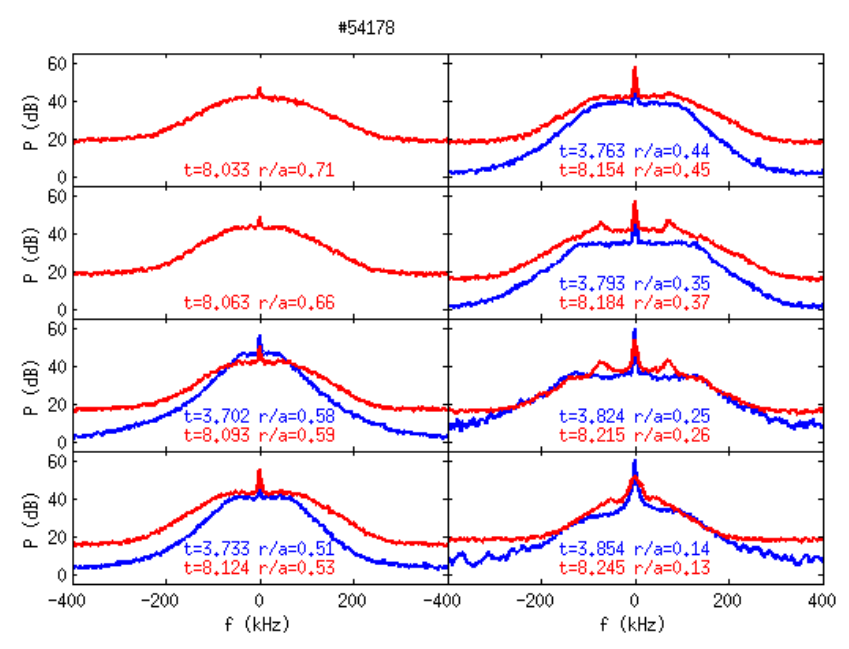

FIG. 5: Reflectometry spectra (Welch with 1024 points averaged over $25 \mathrm{~ms}$ ) at $\mathrm{t}=3.7 \mathrm{~s}$ (Ohmic blue) and $\mathrm{t}=8 \mathrm{~s}$ (LHCD phase red)

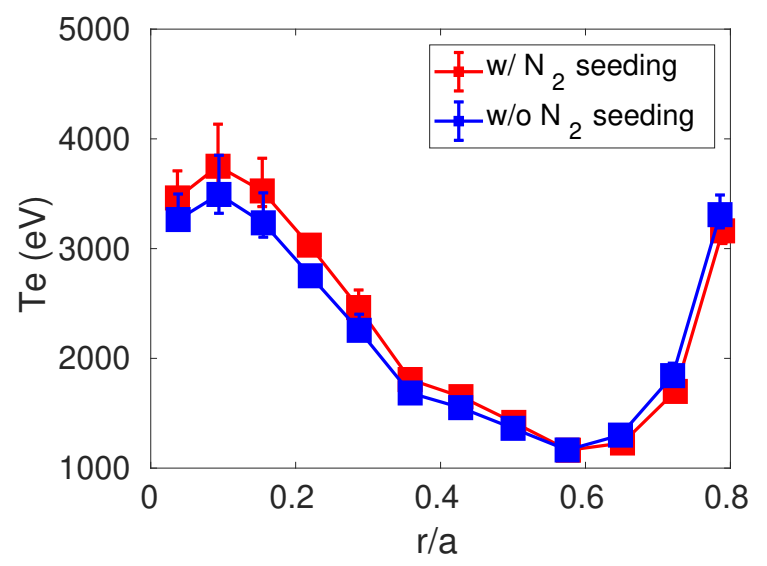

FIG. 6: Electron temperature profiles from ECE measurements with and without nitrogen seeding (phases at 8 and $15 \mathrm{~s}$ respectively). Suprathermal electrons at $r / a>0.6$ are observed during these LHCD heated phases.

consumption during the ohmic phase (up to $4 \mathrm{~s}$ ). In this phase, $Z_{\text {eff }}$ is adjusted until, using the Sauter resistivity [37], the reconstructed loop voltage matches the measured one (see Fig. 9). With this method, $Z_{\text {eff }}$ is found to be 2.8 on the current flat top before LHCD.

The density of light impurities $\mathrm{C} / \mathrm{O} / \mathrm{N}$ is simplified and only $\mathrm{N}$ is assumed to be present. Some $\mathrm{Cu}$ is also known to be present in the discharge thanks to the UV measurements [38] which are not calibrated. Hence, a ratio $n_{C u} / n_{N}=0.04$ is assumed. The amount of $\mathrm{W}$ (density profiles chosen to have the same shape as $n_{e}$ ) is adjusted to match the total radiated power. In METIS, radiated power and charge states of $\mathrm{W}$ increase selfconsistently with the $T_{e}$ profile using ionisation and recombination rates from ADAS data (no transport is assumed). The radiated power from line emission and thermal Bremsstrahlung are computed from the cooling rates. 


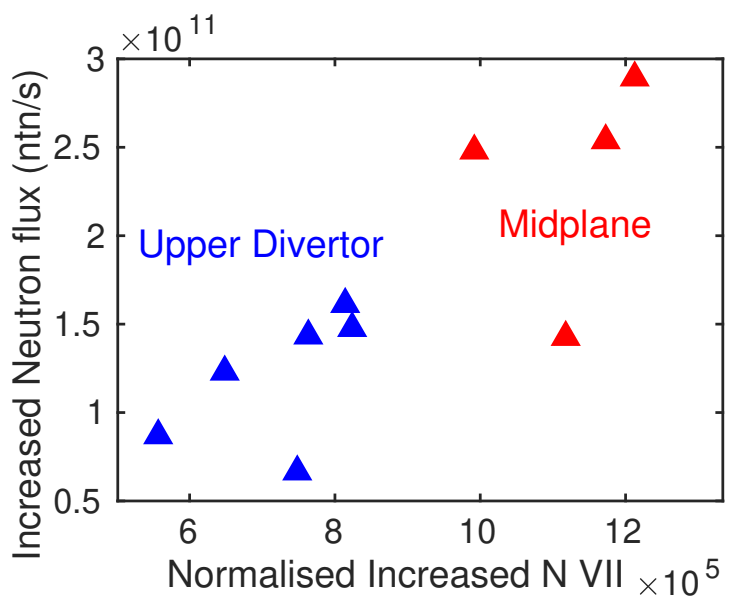

FIG. 7: Increment in the DD neutron rate between phases with and without $N_{2}$ seeding versus increased line emission intensity of N VII normalized to the electron density. $N_{2}$ seeded from the upper divertor/midplane are shown in blue/red.
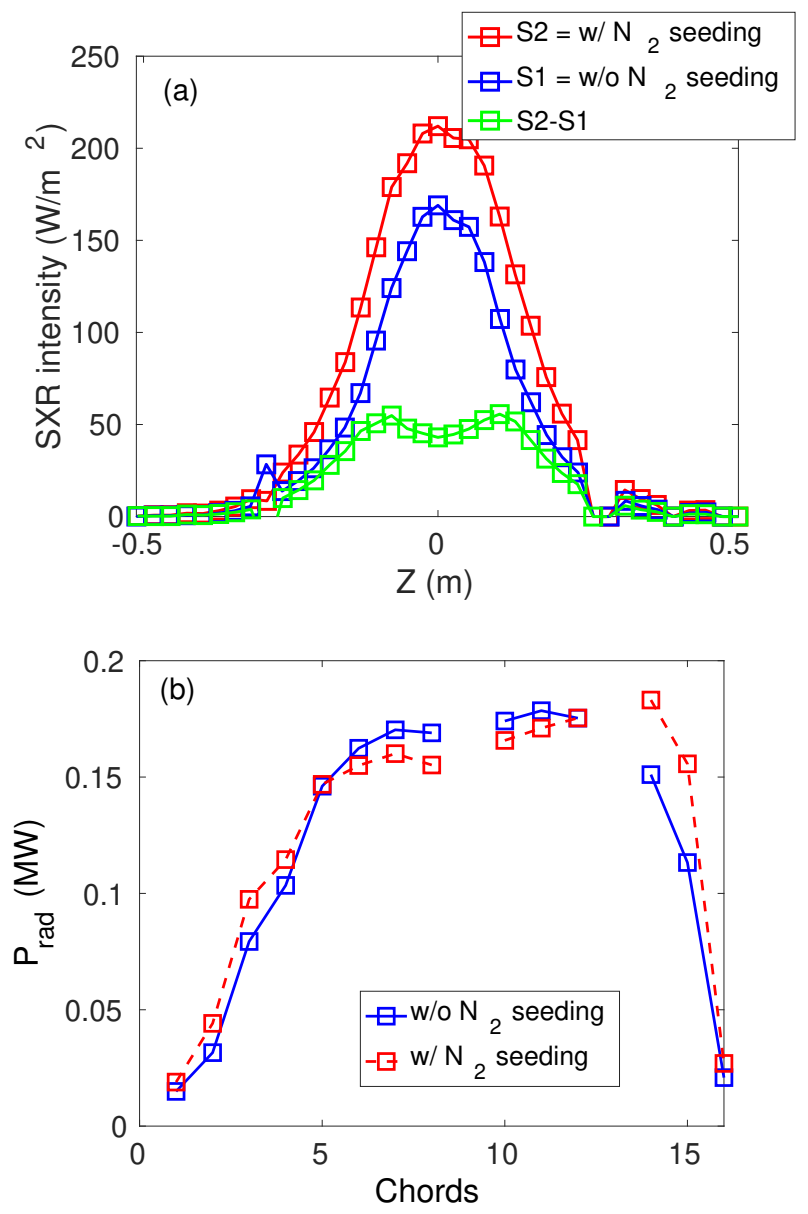

FIG. 8: (a) The horizontal line integrated SXR emission and (b) radiated power for each chords of the two bolometric cameras. Chord 1 starts below the midplane, close to the lower divertor and chord 16 is the furthest view above the midplane, cf Fig. 2.

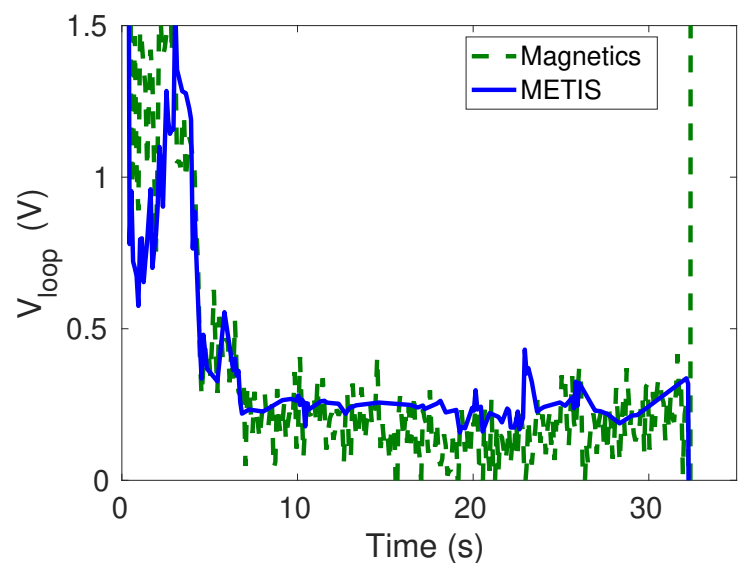

FIG. 9: Time trace of the loop voltage measured by the magnetics compared to the modelled loop voltage of the METIS interpretative run for the pulse \#54178

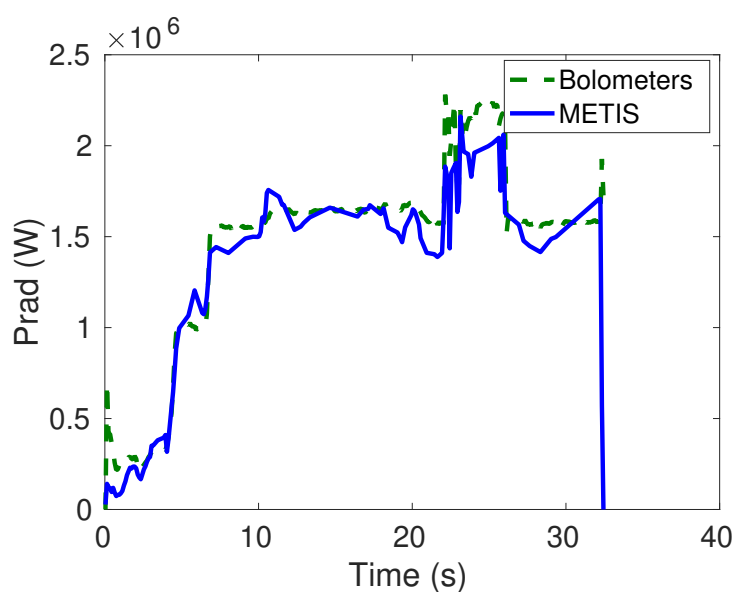

FIG. 10: Radiated power measured by the bolometer vs the modelled radiated power from the interpretative METIS run for \#54178.

As illustrated in Fig. 10, the $\mathrm{W}$ concentration is adjusted during LHCD and LHCD+ICRH phases in order for the METIS reconstructed radiated power to match the measured bolometer value. This leads to $n_{W} / n_{e}=1.1 \times 10^{-4}$ per MW of LHCD and $2 \times 10^{-4}$ per MW of ICRH power.

The $\mathrm{H}$ concentration is measured using the $H_{\alpha} /\left(H_{\alpha}+\right.$ $\left.D_{\alpha}\right)$ ratio from the visible spectroscopy diagnostic [21]. We take $n_{H} /\left(n_{H}+n_{D}\right)=0.1$. The shape of the ion temperature profile is taken to follow the square root of the electron pressure and is scaled with the measured DD neutron rate as illustrated by Fig. 11, yielding $T_{e} / T_{i} \sim$ 2 in the plasma core $(r / a=0.5)$. The assumption we make with regards to the profile shape will be further discussed, as it is particularly important for the power balance analysis.

The thermal energy content based on the ion and electron density and temperature profiles is compared to the energy content obtained by magnetic reconstruction; we 


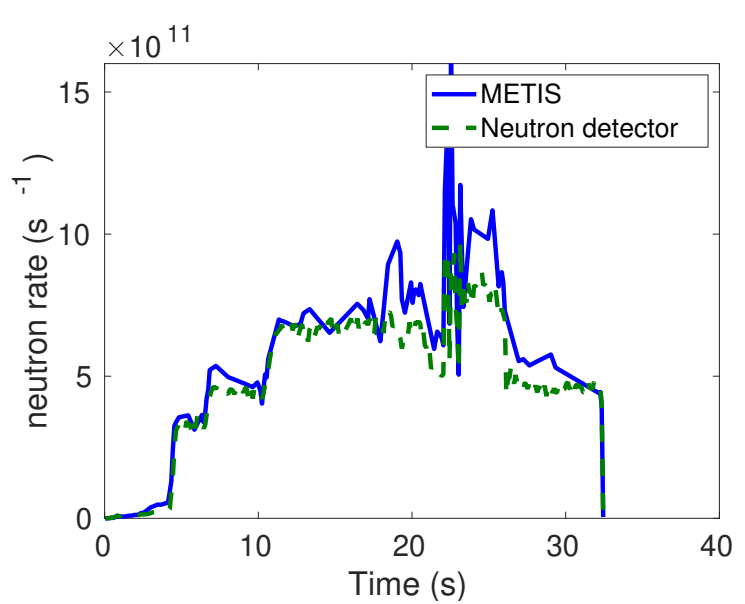

FIG. 11: DD neutron rate as measured vs modelled from the METIS interpretative run for \#54178.

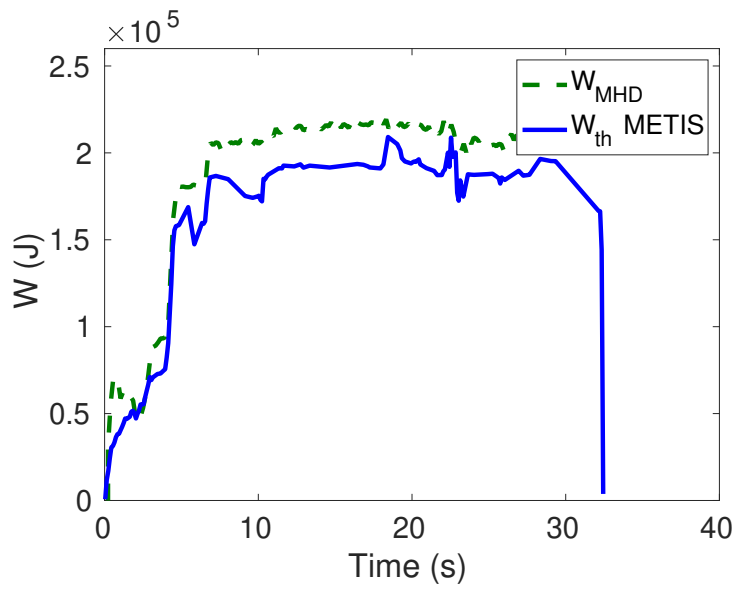

FIG. 12: Energy content estimated from the NICE equilibrium reconstruction and the modelled interpretative METIS thermal energy content for \#54178.

use NICE [39] to perform the construction based on both magnetic measurements and the polarimetry chords. As illustrated on Fig. 12, METIS reconstructed thermal energy content agrees within $20 \%$ with the NICE $W_{\text {MHD }}$.

Modelling work of the LHCD power deposition is ongoing using C3PO-LUKE [40], and thus is not available for this discharge. The difficulty in reproducing the centrally peaked Hard X-Ray measured profile and the measured plasma current is likely due to the presence of heavy $\mathrm{W}$ ions. The modelled current varies from 300 when we assume fully ionised $\mathrm{W}$ ions to $400 \mathrm{kA}$ when using ADAS for $\mathrm{W}$ ionization states. The truth lies somewhere in between these two extremes as electrons of $10 \mathrm{keV}$ or $100 \mathrm{keV}$ will not be impacted by the same ionization state of $\mathrm{W}$. This phenomenon is presently under study. Meanwhile, a slightly off-axis LHCD power deposition has been assumed, at $\rho=0.2$, with a Gaussian width of 0.3 and a current drive efficiency of $5 \times 10^{18} \mathrm{~A} / \mathrm{W} / \mathrm{m}^{2}$.

In the following, we describe a METIS interpretative run at $8 \mathrm{~s}$ during the LHCD heating phase and prior to the $N_{2}$ seeding; this serves as input for the microstability and neoclassical modelling presented in the next Section. In Fig. 13 the ion and electron temperature profiles are plotted together with the electron and ion heat fluxes profiles while taking into account the power deposition from LHCD, the ohmic power, the collisional equipartition and the radiated power. We find the Ware pinch (also shown) to be negligible for density peaking predictions at mid-radius and important in the central radial domain $r / a<0.3$.

It is to be noted that the ratio $Q_{e} / Q_{i}$ at mid-radius is sensitive to the $T_{i}$ profile since the only source for the ion heat flux is the collisional equipartition. Since $T_{i}$ is not measured, we have tested several shapes (proportional to $n_{e}$ or $T_{e}$ ) of the ion temperature profiles while keeping the central value to match the measured neutron flux. The resulting values of $Q_{e} / Q_{i}$ lie between 3 and 4 . Thus, despite the lack of $T_{i}$ measurements, $Q_{e} / Q_{i}$ is expected to be large at mid-radius.

Important parameters for the following analysis at mid-radius are summarized in Table I.

\begin{tabular}{|c|c|c|c|c|c|c|c|c|}
\hline \hline$r / a$ & $R / L_{T_{e}}$ & $T_{e} / T_{i}$ & $\hat{s}$ & $q$ & $Z_{\text {eff }}$ & $\nu_{*}$ & $Q_{e}\left(\mathrm{~kW} / \mathrm{m}^{2}\right)$ & $Q_{i}\left(\mathrm{~kW} / \mathrm{m}^{2}\right)$ \\
\hline 0.5 & 15 & 1.8 & 0.9 & 1.7 & 2.8 & 0.5 & 50 & 13 \\
\hline
\end{tabular}

TABLE I: Parameters for \#54178 at mid-radius taken at $8 \mathrm{~s}$.

\section{THEORETICAL MODELLING}

The turbulent transport is modelled using the gyrokinetic code GKW [1] and results are compared to QuaLiKiz [15] due to its fast computation capabilities and robustness with respect to integrated modelling results in JETTO for JET plasmas [41-43]. The analysis is carried out at $8 \mathrm{~s}$ in the LHCD heating only phase. In these TEM dominated plasmas, characterised by large $T_{e} / T_{i}$ and $Q_{e} / Q_{i}$ (see Table I and density fluctuation measurements in Fig. 5), the limitations of QuaLiKiz due to the assumed fluid eigenfunctions [44] and the reduced collision operator are studied. We investigate the electron particle transport as well as $\mathrm{W}$ transport, then we discuss the impact of $N_{2}$ seeding on confinement.

\subsection{Simulation setup}

For the neoclassical transport, the code NEO is used with 5 Laguerre and 17 Legendre polynomials together with the full Fokker-Planck collision operator. For the turbulent transport, GKW results were not used to benchmark the results of QuaLiKiz but to provide more realistic simulations. For instance, in GKW we use the Miller geometry [45] instead of the $s-\alpha$ geometry as well 

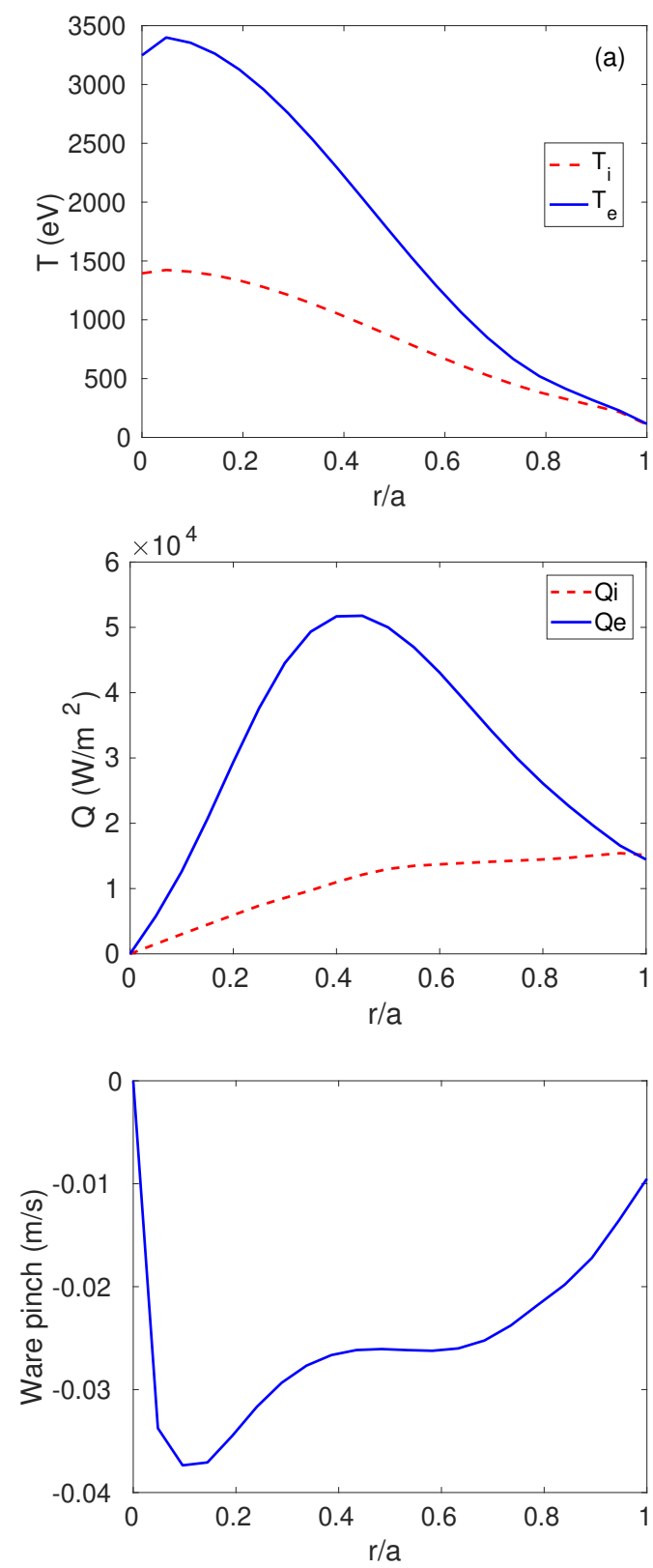

FIG. 13: The profiles of (a) main ion and electron temperature, (b) main ion and electron heat flux and (c) Ware pinch. All these profiles are computed in METIS.

as the full linearized Landau collision operator including an ad-hoc field particle component to ensure energy and momentum conservations; we also include electromagnetic effects with $\beta=0.1 \%$. For the quasilinear flux estimates, the poloidal wave number spectrum used is $k_{\theta} \rho_{i}=[0.2,0.3,0.4,0.5,0.7,0.9,1.1,1.4,1.7,2]$ with $\rho_{i}=m_{i} v_{t h, i} /(e B)$ and the radial wave number taken at $k_{x} \rho_{i}=0$. The quasilinear weight is given by $\gamma /<k_{\perp}^{2}>$ in both GKW and QuaLiKiz, with the operator $\langle\cdots\rangle$ being an average over the mode structure along the field line. The quasilinear heat and particle fluxes do not peak below $k_{\theta} \rho_{i}=0.3$ in the simulations considered here, which validates the range of poloidal wave numbers used. The saturation amplitude is not necessary to assess the electron density peaking since it is determined by the zero flux condition (no central particle source and negligible Ware pinch at mid-radius). On the other hand, the shape of the spectra given by the quasilinear weight is important to properly account for positive and negative contributions to the particle flux at each poloidal wave number.

\subsection{Electron density peaking predictions}

It is observed from interferometry inversions and reflectometry measurements that a peaked electron density profile develops (Fig. 3(b)) in these WEST L-modes. Such peaked profiles are unfavourable regarding $\mathrm{W}$ transport as they contribute to the neoclassical inward convection [46]. Hence, we first investigate the theoretical modelling of such plasmas, particularly the peaked electron density profiles.

In Fig. 14, the quasilinear ratio of the electron heat flux to the ion heat flux $Q_{e} / Q_{i}$ is computed with GKW at mid-radius for a range of normalised density gradients $R / L_{n}$ and ion temperature gradients $R / L_{T_{i}}$ spanning both the ITG (large $R / L_{T_{i}}$ ) and TEM (large $R / L_{n}$ ) domains. The choice of these two parameters is motivated by the absence of $T_{i}$ measurement (and the assumption made on the profile shape, central $T_{i}$ being constrained by the DD neutron flux) and the computation of the zero particle flux condition. All other relevant quantities are set to the values given in Table I. The $\Gamma_{e}=0$ condition, which gives the electron density peaking factor, is shown for each $R / L_{T_{i}}$ value. This condition is always given by a compensation between the turbulent diffusion and convection, i.e. $\Gamma_{e}=0$ is comprised between negative and positive flux regions [47]. As $R / L_{n}$ decreases along the $\Gamma_{e}=0$ contour, $Q_{e} / Q_{i}$ increases due to the stabilisation of ITG modes. The GKW ratios $Q_{e} / Q_{i}=3$ and $Q_{e} / Q_{i}=5$ (to account for assumptions on the LHCD power deposition and on the collisional equipartition) have been plotted over the underlying graph. It is found that these ratios intersect the $\Gamma_{e}=0$ line at respectively $R / L_{n}=5.8$ and $R / L_{n}=5.2$, which is in quantitative agreement with experimental measurements giving an electron peaking factor at mid-radius of $R / L_{n} \sim 5.5$. These peaked density profiles predictions with quasilinear simulations using GKW are also robust with respect to changes in $T_{i}$ at mid-radius and corresponding changes in $T_{e} / T_{i}$. Indeed similar 2D scans as in Fig. 14 were also performed at $T_{e} / T_{i}=1.2$ and 2.3 and similarly peaked density profiles were obtained with $R / L_{n} \sim 6$. This shows that despite the assumptions made on the ion temperature profile at mid-radius, quantitative predictions of the core density peaking factor at this radial location can be achieved.

The particular regime in which these WEST L-mode plasmas are, that is, dominant electron heating with 


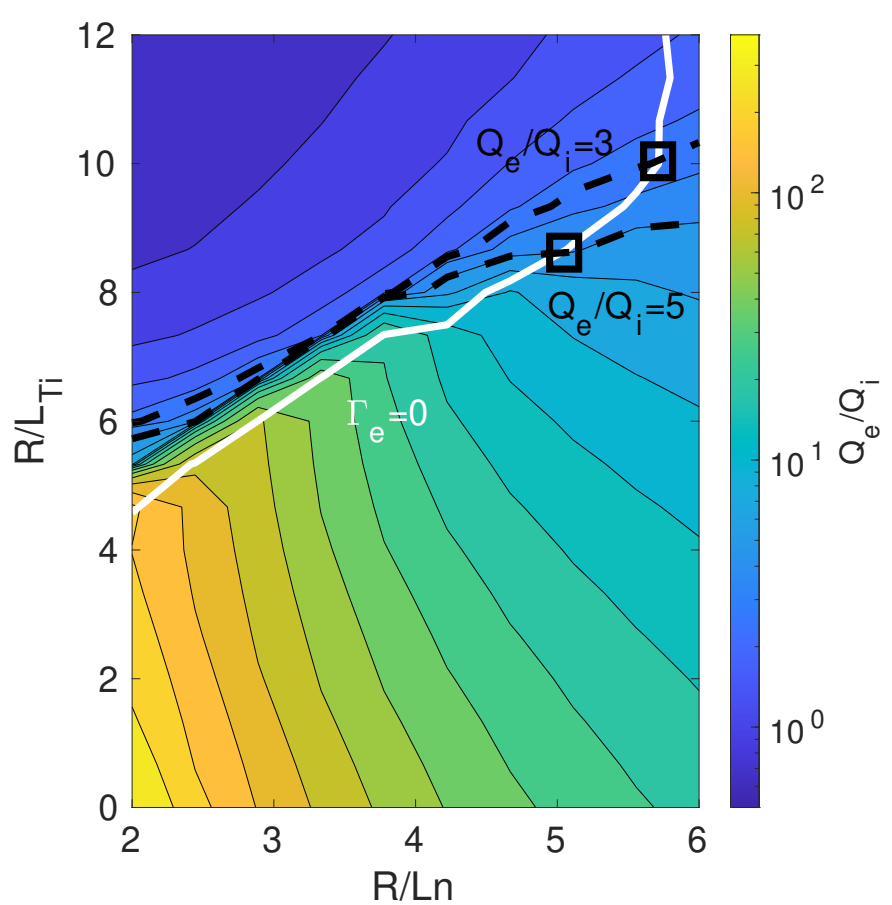

FIG. 14: Quasilinear $Q_{e} / Q_{i}$ computed from GKW. The $\Gamma_{e}=0$ condition (white line) and the $Q_{e} / Q_{i}=3-5$ (black lines) are also shown. Intersections of these conditions are underlined with black rectangles.

$T_{e} / T_{i} \sim 2$ and electron-electron collisionalities of the order of $\nu_{e e} a / v_{t h, i}=0.06$ at mid-radius, can be difficult for reduced models due to the combination of strong TEM turbulence and collisional stabilisation. In Fig. 15 (top panel) the $\Gamma_{e}=0$ conditions are compared between previous results obtained with GKW and simulations performed with QuaLiKiz. In contrast to GKW where turbulence is found on the whole $\left(R / L_{T_{i}}, R / L_{n}\right)$ domain, stable regions are found in QuaLiKiz for $R / L_{T_{i}}<5$ and $R / L_{n}<5$. This yields two regimes regarding $\Gamma_{e}=0$, one at $R / L_{T_{i}}>5$, where turbulent convection compensates turbulent diffusion but gives reduced electron peaking factors $\left(R / L_{n} \sim 2\right)$ compared to $\operatorname{GKW}\left(R / L_{n} \sim 5\right)$ and one regime at $R / L_{T_{i}}<5$ where the electron peaking is given by a threshold for the trapped electron mode turbulence destabilised by $R / L_{n}$. This threshold results in large peaking factors $4<R / L_{n}<6$ due to strong collisional stabilisation of the $\nabla T_{e}$ driven TEM. In both regimes, large discrepancies exist between GKW and QuaLiKiz predictions of the density peaking at $\Gamma_{e}=0$. Additionally, the ratios $Q_{e} / Q_{i}=3-5$ in QuaLiKiz are obtained for larger $R / L_{n}$ and there are no overlapping between $\Gamma_{e}=0$ and $Q_{e} / Q_{i}=3-5$ conditions. These results do not depend only on $R / L_{T_{i}}$ and $R / L_{n}$ and several key parameters have been tested such as $R / L_{T_{e}}, T_{e} / T_{i}$, $\nu_{e i}, \hat{s}, q$. No combinations of these inputs could reconcile the two conditions for the particle flux and the heat fluxes ratio computed with QuaLiKiz. A very strong de-
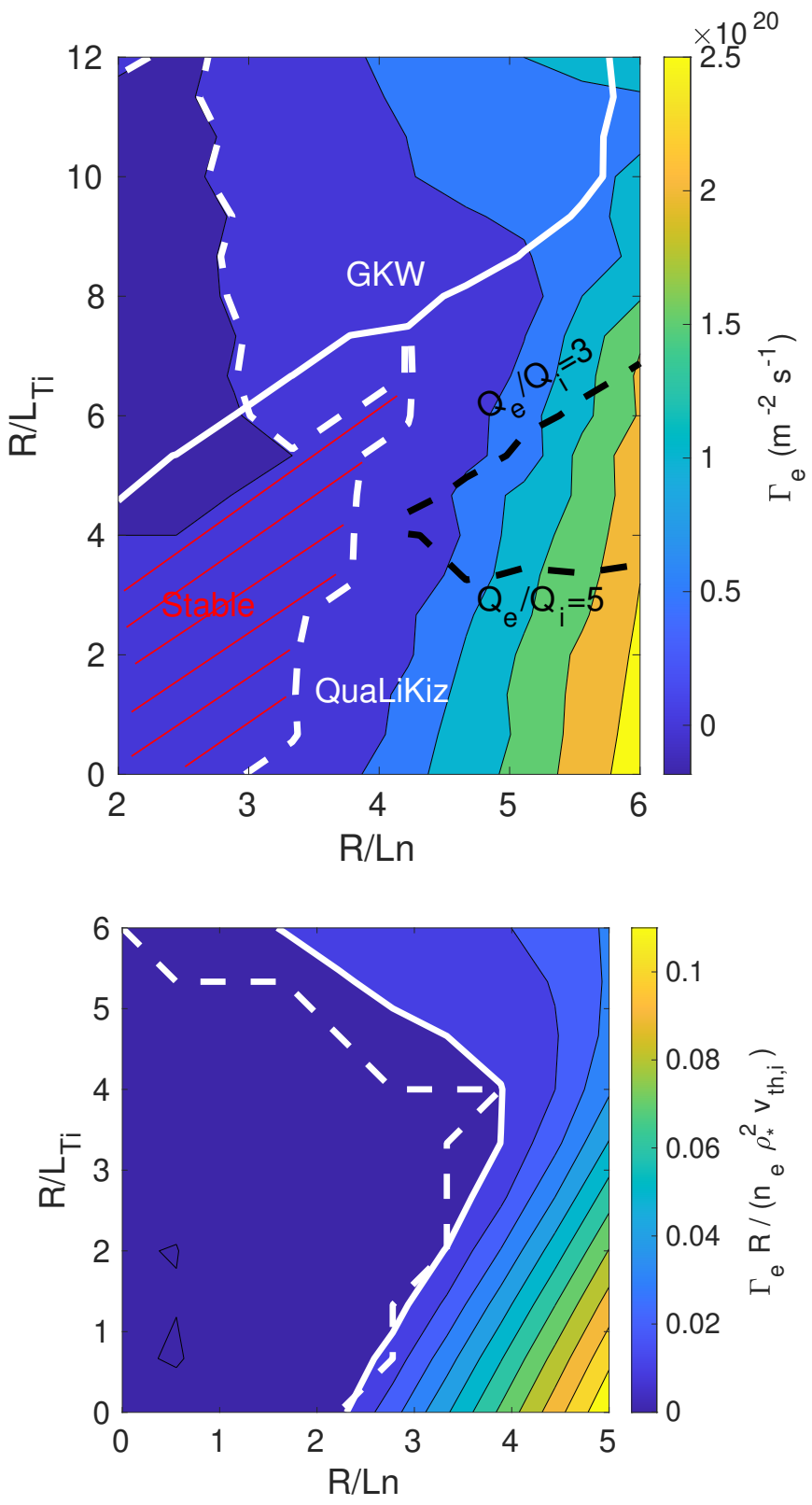

FIG. 15: Comparison of the $\Gamma_{e}=0$ condition between GKW (solide lines) and QuaLiKiz (dashed lines) for the nominal case (top panel) and the simpler case $R / L_{T_{e}}=0$ and $\nu_{e, i}=0$ (bottom panel).

pendence of the turbulent fluxes was found with the collisionality, resulting in overstabilisation of the trapped electron modes destabilised by $R / L_{T_{e}}$ which is not the case in GKW and thus explaining the absence of stable regions in Fig. 14.

This discrepancy between QuaLiKiz and GKW is specific to the TEM dominant condition of the plasmas analysed. Indeed, if we zero $R / L_{T_{e}}$ and $\nu_{e, i}$, we recover a good agreement between the codes as illustrated in Fig. 15 bottom panel. It is worth mentioning that, without $R / L_{T_{e}}$ driven turbulence, the electron peaking factor is 


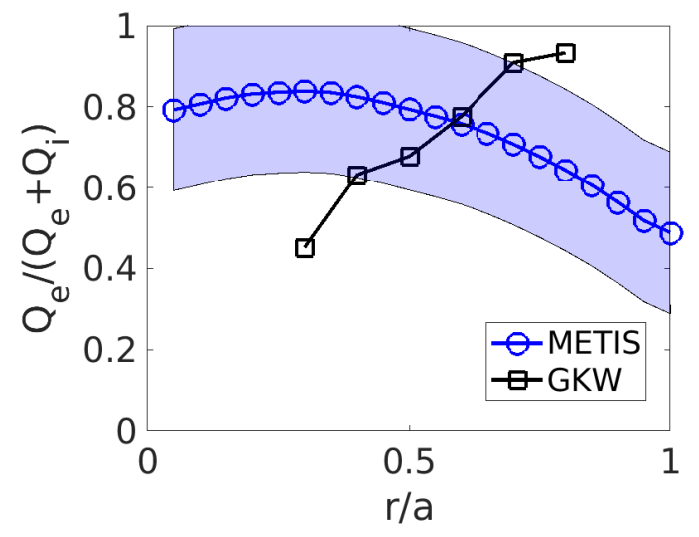

FIG. 16: Ratios of the electron and total heat fluxes computed from GKW. These are compared to results obtained from power balance analysis in the transport code METIS. Inside $r / a=0.3$, linear modes are stable.

given by a transition from a stable and an unstable region in both codes.

From now on, turbulent transport will be modelled with GKW. Nevertheless, it has to be emphasised that such gyrokinetic codes cannot be routinely used in flux driven integrated modelling due to time consuming simulations. First principle based reduced models such as QuaLiKiz are, on the other hand, particularly suited for such endeavors and thus are currently being further validated/developed (in particular regarding the collision operator) for plasma scenario predictions in these TEM dominated regimes [48].

\subsection{Tungsten transport modelling}

Compared to the previous Section where we spanned a large range of $R / L_{T_{i}}$ and $R / L_{n}$, we compute the tungsten transport from the experimental $\left(T_{e}, n_{e}\right)$ and reconstructed profiles (e.g. $T_{i}$ ) with their corresponding gradients (gradient driven approach) on the radial domain $r / a=0.1-0.8$. As a consistency check, the ratio of the electron heat flux over the total heat flux is compared between GKW and the power balance analysis from the transport code METIS (Fig. 16).

From $r / a=0.4$ to $r / a=0.8$, a dominant electron heat flux ratio is qualitatively reproduced in the modelling. Inside $r / a=0.2$ linear modes are found stable. We note that in this region, micro-tearing modes, which can drive large electron heat fluxes [49], are linearly marginally stable. GKW flux ratios decrease towards the magnetic axis, unlike the experimentally inferred fluxes. This can be attributed to missing MHD transport such as sawteeth (experimentally observed) in GKW modelling but also to the assumed LHCD power deposition profile. Aside $r / a=0.3$, dominant TEM turbulence is found in the simulations yielding realistic ratios of $Q_{e} / Q_{t o t}>0.5$ for $r / a$ above 0.3 . Observation of quasi-coherent modes from $r / a=0.26$ to $r / a=0.45$ also supports the prediction of TEM instabilities.

We now analyse tungsten transport with these conditions. To account for neoclassical tungsten transport, the turbulent amplitude from GKW has been normalised using the turbulent ion heat flux from power balance analysis (as in e.g. $[50,51]$ ). The total tungsten flux can then be expressed as:

$$
\Gamma_{\mathrm{W}}=\Gamma_{\mathrm{W}, \mathrm{NEO}}+\Gamma_{\mathrm{W}, \mathrm{GKW}} \frac{Q_{\mathrm{i}, \mathrm{an}}}{Q_{\mathrm{i}, \mathrm{GKW}}}
$$

with the subscript 'an' denoting the anomalous transport computed from the power balance ion heat flux $Q_{\mathrm{i}, \text { an }}=Q_{\mathrm{i}, \mathrm{PB}}-Q_{\mathrm{i}, \mathrm{NEO}}$. For a stationnary phase, without tungsten sources inside $r / a=0.8$, the resulting tungsten peaking factor can be computed with:

$$
R / L_{n_{W}}=-R \frac{V_{\mathrm{NEO}} / Q_{\mathrm{i}, \mathrm{an}}+V_{\mathrm{GKW}} / Q_{\mathrm{i}, \mathrm{GKW}}}{D_{\mathrm{NEO}} / Q_{\mathrm{i}, \mathrm{an}}+D_{\mathrm{GKW}} / Q_{\mathrm{i}, \mathrm{GKW}}}
$$

In the top panel of Fig. 17 the different transport coefficients from Eq. 1 are summarised with no toroidal rotation velocity $V_{\phi}=0 \mathrm{~km} / \mathrm{s}$. In the radial domain where turbulence is linearly destabilised $(0.3 \leq r / a \leq 0.8)$, the neoclassical tungsten convection and diffusion coefficients are 3 orders of magnitude lower than the corresponding turbulent coefficients. For $r / a<0.3$, linear turbulence is stable and thus neoclassical transport of tungsten, eventhough relatively small, is the dominant transport mechanism.

In the bottom panel of Fig. 17, the resulting tungsten peaking factor (normalised density gradient for $\Gamma_{W}=0$ ) can be separated into two regimes. Inside $0.3 \leq r / a \leq$ 0.8 , the tungsten peaking factor is defined solely by turbulent transport coefficients (neoclassical couterparts are negligible) and remains below the electron density peaking. Turbulent diffusion tends to flatten the $\mathrm{W}$ profiles resulting in a peaking factor of $\sim 2$ at mid-radius compared to an electron peaking factor of $\sim 5.5$. Below $r / a<0.3$, neoclassical transport determines the $\mathrm{W}$ peaking factor resulting in larger values compared to $R / L_{n_{e}}$. Nevertheless, neoclassical peaking of $R / L_{n_{W}}$ remains at values much lower than what is encountered in tungsten accumulation scenarios (e.g. [4]).

The impact of toroidal rotation on central neoclassical tungsten transport is key to explaining large neoclassical convections and the resulting accumulation [4, 12]. In this context the same simulations as in Fig. 17 have been performed including a toroidal rotation profile with a central value of the toroidal velocity $V_{\phi}(r / a=0)=150$ $\mathrm{km} / \mathrm{s}$, decreasing to 0 at $r / a=1$. These profiles have been included for both neoclassical and turbulent transport and the results are gathered in Fig. 18 (top panel). With toroidal rotation, neoclassical convection is increased and is now up to one order of magnitude larger than the rescaled turbulent convection. Neoclassical diffusion, on the other hand, remains lower than the turbulent diffusion but only 1 order of magnitude instead of 3 

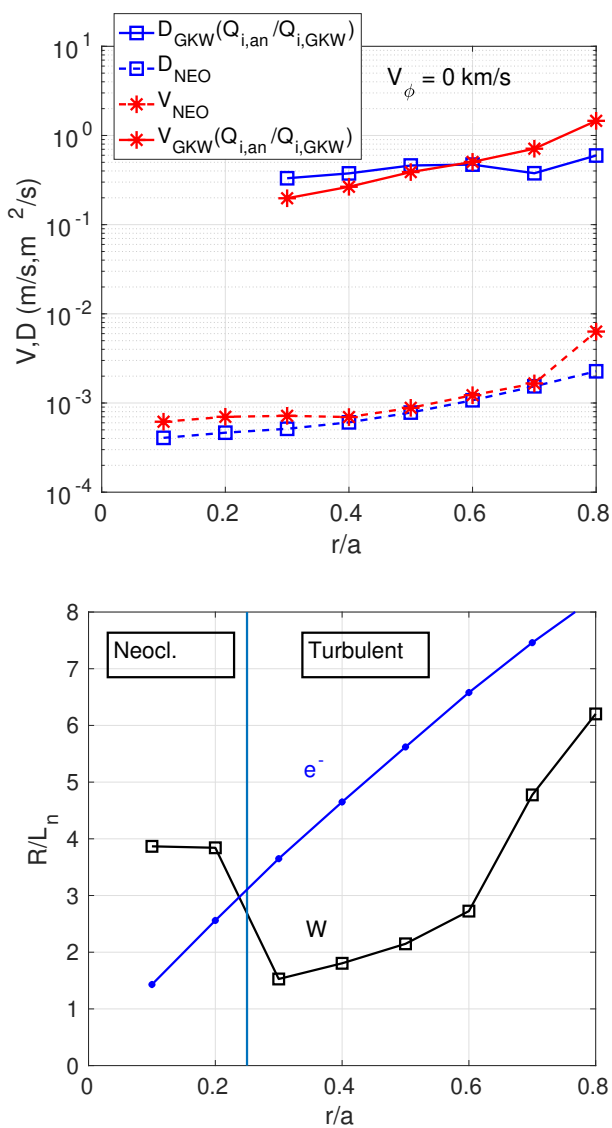

FIG. 17: Turbulent vs neoclassical transport coefficients (top panel) using the scaling factor as discussed in the text. The resulting tungsten peaking factor is shown in the bottom panel.

as in Fig. 17. Additionally, it can be noted that the neoclassical diffusion increases as $r / a$ decreases which comes from the peaked toroidal rotation profile used. Finally, in Fig. 18, the turbulent convection is decreased compared to the case without toroidal rotation due to the additional roto-diffusion mechanism with centrifugal effects [52]. Even in the absence of torque generated by NBI heating systems, intrinsic rotation can be substantial. To include such levels of rotation in the analysis (not measured in this discharge), an additional case with a central toroidal rotation of $20 \mathrm{~km} / \mathrm{s}$ has been added. This value is based on previous measurements performed on the Tore Supra tokamak in ohmic [53, 54] and LHCD heated phases [55]. The resulting tungsten peaking factor for the two chosen levels of rotation is now larger (Fig. 18 bottom panel) in the whole radial domain but more substantially inside $r / a<0.3$ due to stable turbulence and dominant neoclassical transport. This increase of $R / L_{n_{W}}$ is due to the neoclassical convection increasing with toroidal rotation. In the case of central $V_{\phi}=150$ $\mathrm{km} / \mathrm{s}$, similar values of $R / L_{n_{W}}$ as observed in tungsten accumulation phases at AUG, (e.g. [5]) or JET (e.g. [4]) are found. For the intrinsic rotation case, a more modest increase in $R / L_{n_{W}}$ is found (a factor 5 lower than for
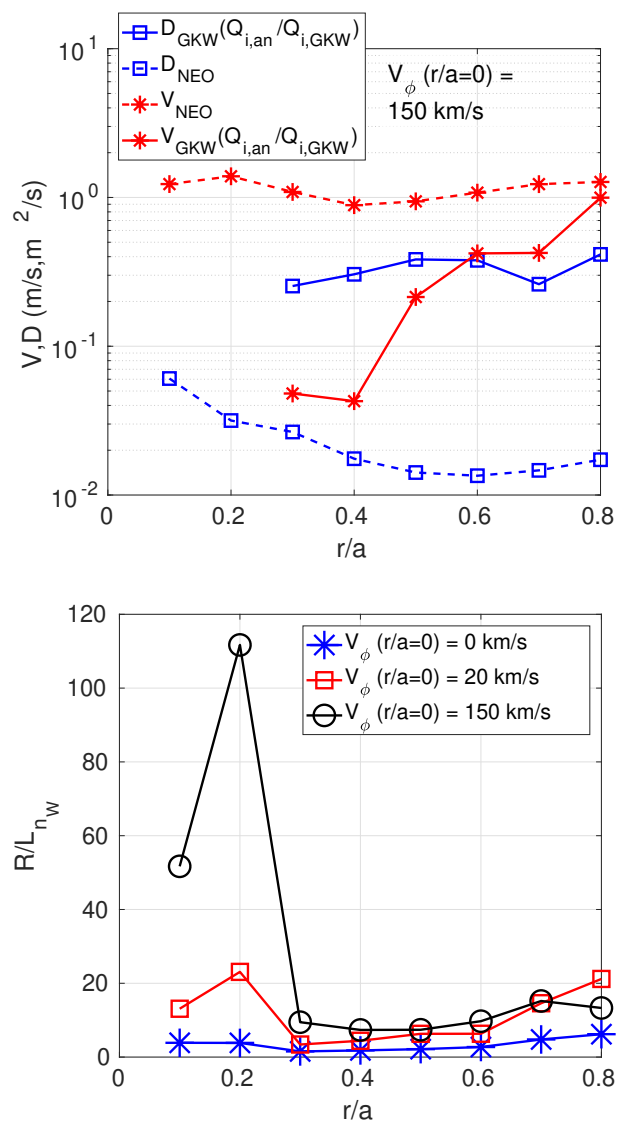

FIG. 18: Same as Fig. 17 including now a toroidal rotation profile. The resulting $R / L_{n_{W}}$ is also shown for different levels of central toroidal rotation velocities (these values are discussed in the text).

$\left.V_{\phi}(r / a=0)=150 \mathrm{~km} / \mathrm{s}\right)$ but still noticeable. In the turbulence dominated radial domain $0.3 \leq r / a \leq 0.8$, the increased neoclassical convection is tempered by the turbulent diffusion. Nevertheless, W peaking factors in this domain are larger than for a pure turbulent case.

In this WEST plasma the observed absence of $\mathrm{W}$ accumulation can be theoretically explained by the absence/reduced level of toroidal rotation and the large turbulent diffusion allowing for low $\mathrm{W}$ neoclassical transport inside $r / a=0.3$ and leading to a dominant turbulent transport for $r / a>0.3$ as is also the case in Alcator C-Mod ICRH heated H-mode plasmas [13]. Even for the peaked density profiles found in these WEST L-mode plasmas (which should drive unfavourable $\mathrm{W}$ convection) compared to the flat profiles found in [13], one of the main parameters for central tungsten accumulation is the central toroidal velocity. In our fixed gradients approach, the role of the central particle source due to NBI heating systems is not considered and could also have a significant impact $[14,42]$. Overall, the resulting $\mathrm{W}$ density gradient lengths are lower/comparable to the electron density gradient lengths accross most of the plasma core. 


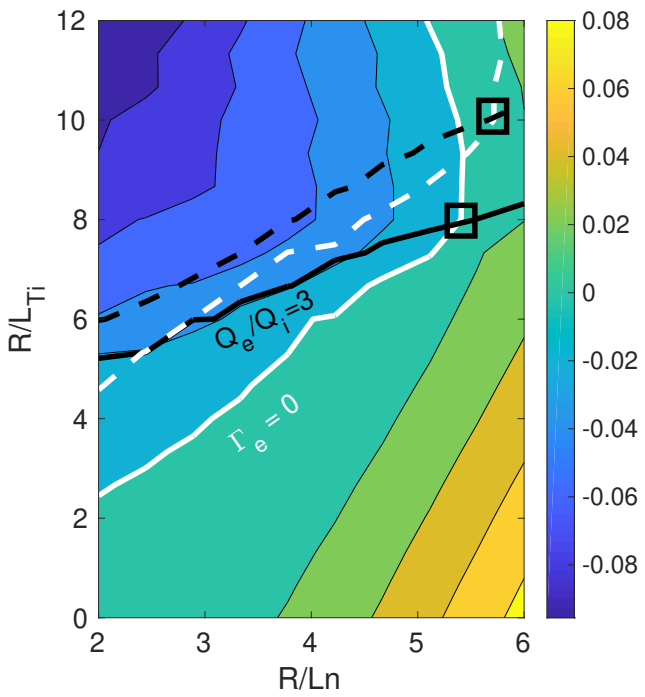

FIG. 19: The normalised electron flux computed from $\operatorname{GKW}\left(\Gamma_{e}=\right.$ 0 condition in white) is compared for a pure plasma (solid lines) and for a contaminated plasma including nitrogen $\left(n_{N} / n_{e}=0.02\right)$, copper and tungsten (dashed lines). The latter two impurities almost behave as traces due to low concentrations $\left(n_{z} / n_{e} \sim 10^{-4}\right)$. Contours correspond to the pure plasma case.

\section{4. $N_{2}$ impact on stability and $\mathrm{W}$ transport}

As analysed in Section 2, it can be seen that the nitrogen seeding improves the plasma confinement and the neutron flux. To study this behaviour, simulations in the $2 \mathrm{D}$ domain $\left(R / L_{T_{i}}, R / L_{n}\right)$ of Fig. 14 are performed for a pure plasma (no impurities) at mid-radius and for a plasma including $N_{2}$ to a concentration of $n_{N} / n_{e}=0.02$. On Fig. 19 such results are compared and the $\Gamma_{e}=0$ and $Q_{e} / Q_{i}=3$ conditions are emphasised. It is found that, with $N_{2}, \Gamma_{e}=0$ and $Q_{e} / Q_{i}=3$ are simultaneously achieved for larger $R / L_{T_{i}}$ values; this is in qualitative agreement with the increased DD neutron rate measured in the experiment. The electron peaking factor matching both $\Gamma_{e}=0$ and $Q_{e} / Q_{i}=3$ is not particularly affected, increasing from 5.2 for the pure plasma to 5.8 with nitrogen. This is consistent with the minor impact observed on the experimental electron density profiles during nitrogen seeding phases.

To further investigate the shift observed in $R / L_{T_{i}}$ found in Fig. 19, a scan in the nitrogen concentration only, i.e. while keeping all the other input parameters fixed, has been performed starting from profiles of the $8 \mathrm{~s}$ phase (without nitrogen seeding). The quasilinear heat flux ratios $Q_{e} / Q_{t o t}$ at mid-radius are then plotted in Fig. 20. It is observed that with increasing nitrogen concentrations, the electron heat flux becomes increasingly dominant over the ion heat flux (sum over the main ions and nitrogen). This is consistent with the stabilisation of ITG modes.

The normalised frequencies for the most unstable lin-

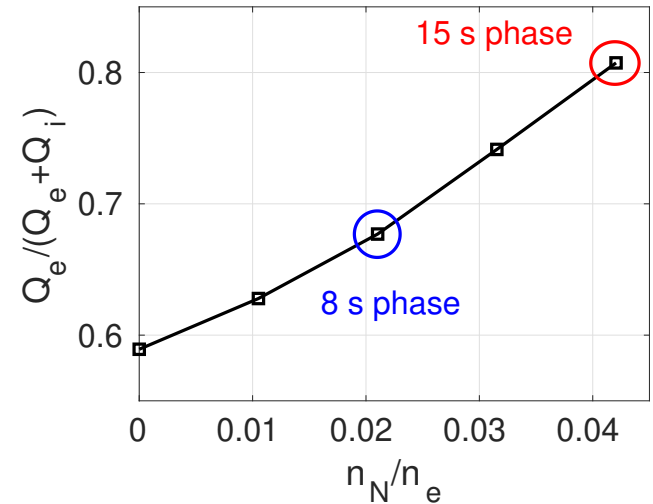

FIG. 20: Predicted ratio of the electron heat flux to the total heat flux (electrons, deuterium, nitrogen) at $r / a=0.5$ with increasing nitrogen concentrations up to levels from the nitrogen seeding phase at $15 \mathrm{~s}$.

ear modes are plotted (Fig. 21) for the wave number range $k_{\theta} \rho_{i}=0.2-1.5$ at increasing nitrogen concentrations. Negative/positive frequencies correspond to the direction of the electron/ion diamagnetic drifts and are characteristic of TEM/ITG modes. With increasing $n_{N} / n_{e}$, the transition between the TEM and ITG modes is pushed to larger wave numbers, hence contributing less to the radial transport. This clearly shows that ITGs are stabilised compared to TEMs modes, mostly from main ion dilution, resulting in higher $Q_{e} / Q_{t o t}$. For a fixed ion heat flux $Q_{i}$, a larger $R / L_{T_{i}}$ is needed, leading to better confinement and an increased $T_{i}$ (consistent with the observed increased neutron flux during nitrogen seeding phases at WEST). This stabilisation of turbulence and in particular of the ITG has been largely documented in previous theoretical (e.g. [56, 57]) and experimental studies (e.g. [58]). It is to be noted that in our approach, we did not considered the $E \times B$ shearing (no toroidal rotation profile) which could also play an increased role with reduced momentum transport in the $N_{2}$ seeding phase.

In Fig. 3, it was shown that the central $\mathrm{W}$ peaking factor, computed from SXR line intensities [59], is reduced during the nitrogen seeding phase. Thus, $R / L_{n_{W}}$ is computed for the two radial positions $r / a=0.5$ and $r / a=0.1$ where turbulent and neoclassical transport dominate respectively. Fig. 22 shows the tungsten peaking factor against the nitrogen concentrations. At $r / a=0.5$, the turbulent $R / L_{n_{W}}$ is not significantly affected for the discussed range of $n_{N} / n_{e}$. In contrast, $R / L_{n_{W}}$ from neoclassical transport first decreases (increasing neoclassical diffusion) and then increases (increasing neoclassical convection) with $n_{N} / n_{e}$. Finally, the total $R / L_{n_{W}}$ as computed using Eq. 1 is dominated by turbulent transport and thus is not changed with the fraction of nitrogen content. At $r / a=0.1$, neoclassical diffusion increases, with little increase of the convection resulting in a decrease of the tungsten peaking factor. This is in qualitative agreement with observations dis- 


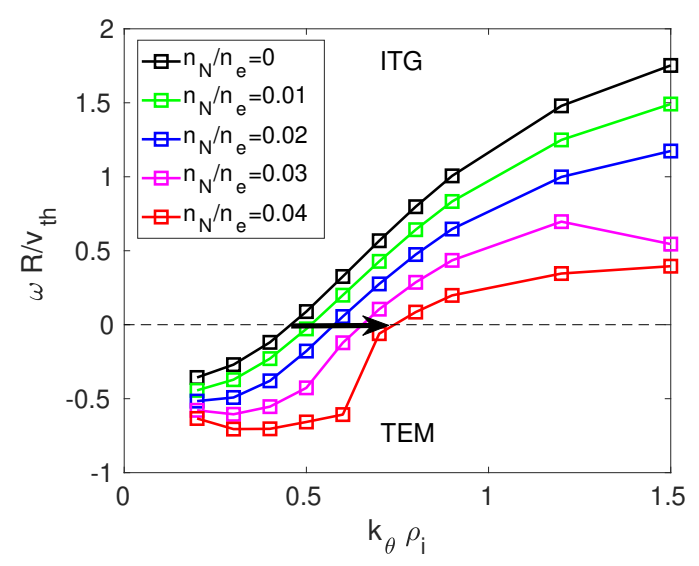

FIG. 21: Normalised linear mode frequency spectra with increasing nitrogen fractions at $r / a=0.5$. Negative values correspond to the electron diamagnetic drift direction.

cussed in Section 2 and can be explained from neoclassical transport only, with increasing nitrogen concentrations.

\section{CONCLUSIONS}

Long pulse dicharges were obtained in the third experimental campaign of the WEST tokamak in a robust and reproducible way. It is observed that tungsten does not centrally accumulate in such discharges eventhough the electron density peaking from these L-mode plasmas are relatively large $\left(R / L_{n} \sim 5.5\right.$ at mid-radius $)$. In the core of the WEST plasmas with dominant radio frequency electron heating, reflectometry measurements feature quasi-coherent modes, signature of trapped electron mode turbulence. Improved confinement is also observed during nitrogen seeding phases where the neutron flux increases with the nitrogen content. The absence of $\mathrm{W}$ accumulation in radio-frequency heated plasmas is not new and it was also observed in Alcator C-Mod H-mode plasmas [13] with ICRH heating only. However, the density profiles in these plasmas are flat which reduces the unfavourable inward neoclassical convection of $\mathrm{W}$.

To explain the features observed in these WEST long pulse L-mode plasmas, extensive neoclassical and turbulent simulations have been performed with the codes NEO and GKW/QuaLiKiz respectively. A particular effort has also been made with regards to the input data for such gradient driven codes. Indeed, interpretative integrated modelling simulations have been performed with METIS to keep a reasonable level of consistency between available measurements and assumptions (e.g. on the $T_{i}$ profiles). WEST operates in dominant electron heating $\left(Q_{e} / Q_{i}>3\right.$ at mid-radius), where TEM are the dominant unstable modes. For this turbulence regime, comparing quasilinear turbulent transport with QuaLiKiz and GKW yielded strong disagreement. In-
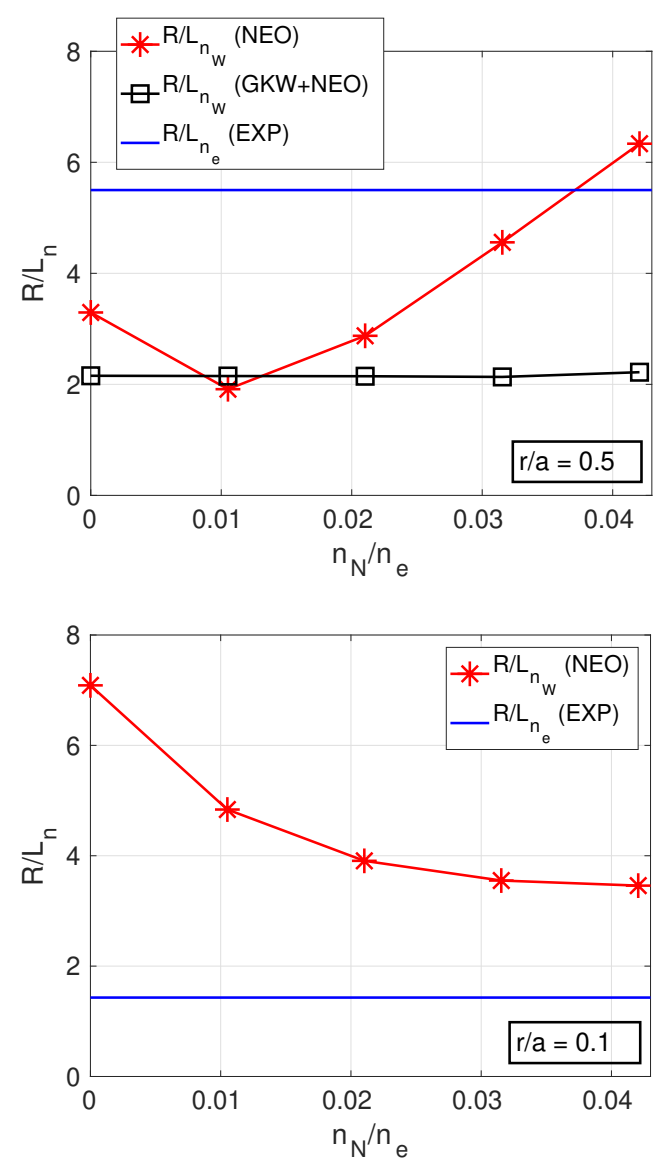

FIG. 22: Tungsten peaking factor including neoclassical and turbulent transport for increasing fraction of nitrogen at $r / a=0.5$ (top panel). The purely neoclassical peaking factor has been also computed at $r / a=0.1$ (bottom panel) for increasing $n_{N} / n_{e}$ where turbulence is completely stabilised in the modelling.

deed, a reduced collision operator as well as the fluid limit for the eigenfunctions are used in QuaLiKiz which overstabilise $R / L_{T_{e}}$ driven TEM. This results in discrepancies with GKW results and experimental observations regarding the electron density peaking and the heat flux ratio $Q_{e} / Q_{i}$. This clearly shows that in regimes with dominant electron heating and non-negligible collisional effect, results obtained from reduced models (in particular for integrated modelling) should be treated with caution. Additional development is undergoing for the QuaLiKiz collision operator to improve predictions in such regimes.

The electron density peaking deduced from GKW simulations and interferometry inversions are in quantitative agreement. The large values of $R / L_{n_{e}}$ are obtained in regimes where TEM turbulence is dominant, which is consistent with observations made at the ASDEX Upgrade tokamak [60]. In this condition, neoclassical convection of tungsten is negligible for $0.3 \leq r / a \leq 0.8$ due to large turbulent diffusion, resulting in marginally peaked predicted $\mathrm{W}$ profiles. For $r / a<0.3$ where turbulence is completely stabilised and neoclassical trans- 
port dominates, the resulting tungsten peaking factor $\left(R / L_{n_{W}}\right.$ at the zero particle flux $\left.\Gamma_{W}=0\right)$ stays below 4. This is due to the absence of significant toroidal rotation in such plasmas, which reduces the unfavourable neoclassical W convection which is in contrast produced by centrifugal effects when NBI heating is used in AUG and JET. The observation of steady core radiated power during these long pulses are consistent with strong turbulent diffusion and reduced neoclassical tungsten convection, preventing $\mathrm{W}$ to accumulate, a result also found in [13]. Finally, the observed improved confinement during nitrogen seeding phases was investigated. As in previous works $[56,57]$, it is found from linear gyrokinetic simulations (GKW) that the linear ITG modes are mainly stabilised from dilution yielding higher $R / L_{T_{i}}$ to match the heat flux ratio $Q_{e} / Q_{i}$; this could explain the observed increase in core confinement.

\section{Acknowledgments}

This work was supported by the program of China Scholarships Council, the National Natural Science Foundation of China (Grant Nos. 11775269, 11905146), the National Key Research and Development Program of China (No. 2018YFE0311100), and Users with Excellence Program of Hefei Science Center CAS (No. 2019HSC-UE014).
[1] A. G. Peeters, Y. Camenen, F. J. Casson, W. A. Hornsby, A. P. Snodin, D. Strintzi, G. Szepezi, Computer Physics Commnunications 180, 2650 (2009)

[2] E. Belli and J. Candy, Plasma Physics and Controlled Fusion 50, 095010 (2008)

[3] E. Belli and J. Candy, Plasma Physics and Controlled Fusion 54, 015015 (2012)

[4] C. Angioni et al, Nuclear Fusion 54, 083028 (2014)

[5] T. Odstrcil et al, Plasma Physics and Controlled Fusion 60, 014003 (2018)

[6] J. Bucalossi et al, Fusion Engineering and Design 89, 907 (2014)

[7] C. Bourdelle et al, Nuclear Fusion 55, 063017 (2015)

[8] M. Richou et al, Fusion Engineering and Design 98-99, 1394-1398 (2015)

[9] T. Dittmar et al, to be published in Physica Scripta

[10] C. Angioni et al, Physics of Plasmas 22, 055902 (2015)

[11] F. Casson et al, Plasma Physics and Controlled Fusion 57, 014031 (2015)

[12] C. Angioni and P. Helander, Plasma Physics and Controlled Fusion 56, 124001 (2014)

[13] A. Loarte et al, Physics of Plasmas 22, 056117 (2015)

[14] F. J. Casson et al, Nuclear Fusion, Accepted for publication (2020)

[15] C. Bourdelle et al, Plasma Physics and Controlled Fusion 58, 014036 (2016)

[16] J. F. Artaud, Nuclear Fusion 58, 105001 (2018)

[17] J. Bucalossi et al, Symposium on Fusion Engineering (2019)

[18] M. Missirlian et al, Fusion Engineering and Design 89, 1048-1053 (2014)

[19] J. Bucalossi et al, Plasma Facing Materials Conference (2019)

[20] M. Goniche et al, Radiofrequency power in Plasmas Conference (2019)

[21] O. Meyer et al, Review of Scientific Instruments 87, 11E309 (2016)

[22] C. Gil et al, Fusion Engineering and Design 140, 81 (2019)

[23] L. Colas et al, Radiofrequency power in Plasmas Conference (2019)

[24] J. L. Ségui et al, Review of Scientific Instruments 76, 123501 (2005)
[25] R. J. Groebner et al, Nuclear Fusion 41, 1789 (2001)

[26] R. Sabot et al, Nuclear Fusion 46, S685 (2006)

[27] A. Krämer-Flecken et al, Nuclear Fusion 44, 1143 (2004)

[28] Y. Sun et al, Review of Scientific Instruments 89, 073504 (2018)

[29] V. A. Vershkov et al, Nuclear Fusion 45, S203 (2005)

[30] H. Arnichand et al, Nuclear Fusion 54, 123017 (2014)

[31] H. Arnichand et al, Plasma Physics and Controlled Fusion 58, 014037 (2016)

[32] M. Osakabe et al, Plasma Physics and Controlled Fusion 56, 095011 (2014)

[33] R. R. Weynants et al, Nuclear Fusion 39, 1637 (1999)

[34] G. L. Jackson et al, Nuclear Fusion 42, 28 (2002)

[35] J. Schweinzer et al, Nuclear Fusion 51, 113008 (2011)

[36] H. Urano et al, Nuclear Fusion 55, 03310 (2015)

[37] O. Sauter et al, Physics of Plasmas 6, 2834 (1999)

[38] C. Desgranges, EPS (2018)

[39] B. Faugeras et al, Nuclear Fusion 58, 106032 (2018)

[40] Y. Peysson and J. Decker Physics of Plasmas 15, 092509 (2008)

[41] J. Citrin et al, Plasma Physics and Controlled Fusion 59, 124005 (2017)

[42] S. Breton et al, Nuclear Fusion 58, 096003 (2018)

[43] F. Casson et al, to be submitted (2019)

[44] P. Cottier et al, Plasma Physics and Controlled Fusion 56, 015011 (2014)

[45] R. L. Miller, M. S. Chu, J. M. Greene, Y. R. Lin-Liu and R. E. Waltz, Physics of Plasmas 5, 973 (1998)

[46] P. Helander and D. J. Sigmar, Collisional Transport in Magnetised Plasmas

[47] C. Angioni et al, Physics of Plasmas 16, 060702 (2009)

[48] C. D. Stephens, private communications

[49] H. Doerk et al, Physical Review Letters 106, 155003 (2011)

[50] C. Angioni et al, Nuclear Fusion 51, 023006 (2011)

[51] F. Casson et al, Nuclear Fusion 56, 063026 (2013)

[52] Y. Camenen et al, Physics of Plasmas 16, 012503 (2009)

[53] A. Romannikov et al, Nuclear Fusion 40, 319 (2000)

[54] J. Bernardo et al, Plasma Physics and Controlled Fusion 57, 035002 (2015)

[55] B. Chouli et al, Plasma Physics and Controlled Fusion 56, 095018 (2014)

[56] M. Fröjdh et al, Nuclear Fusion 32, 419 (1992) 
[57] R. R. Dominguez and G. M. Staebler et al, Nuclear Fusion 33, 51 (1993)

[58] G. McKee et al, Physical Review Letters 84, 1922 (2000)

[59] M. Sertoli et al, Journal of Plasma Physics 85, 905850504
(2019)

[60] C. Angioni et al, Physics of Plasmas 12, 040701 (2005) 